Recibido: 18 de setiembre del 2016

Aprobado: 30 de setiembre del 2016

\title{
Modelo de GESTIÓN DE INCIDENTES PARA UNA ENTIDAD ESTATAL
}

\author{
Alexander Alberto Loayza Uyehara \\ alexloayza@gmail.com \\ DSB Mobile. Lima, Perú
}

Resumen

El presente artículo muestra la elaboración de un modelo de gestión de incidentes basado en ITIL v3.0, que permitirá mejorar la entrega de servicios de tecnologías de información (TI) ofrecidos por la Oficina Nacional de Gobierno Electrónico e Informática (ONGEI). Ante el incremento de los requerimientos en el uso de los servicios de TI por parte de otras entidades del estado (usuarios), se analizó la situación actual de la ONGEI para reconocer sus problemas en la gestión de incidentes, así como las causas que lo originan. De esta manera se identificó que no se abordaban las severidades, los tiempos de respuesta ni los de restauración; y así también se comprobó la existencia de duplicidad de registros. del servicio se propuso un nuevo modelo de gestión y la adquisición de una herramienta (ServiceTonic, elaborado por la empresa del mismo nombre) para gestionar adecuadamente los incidentes y la gestión de servicios.

Palabras clave: gestión de incidentes / ITIL / organismo del estado peruano

\section{Abstract}

This article shows the development of an Incident Management Model based on ITIL v 3.0 that will allow the improvement of information technology services (TI) delivery, provided by the National Office of Electronic Government and Informatic (ONGEI). Due to the increase of requirements in the use of the TI services from other State entities (users), the current situation of ONGEI was analyzed in order to identify their incident management problems as well as the causes that originate them. In this way, it was identified that the severities were not foreseen, neither the response nor the restore times; it was also found the existence of duplication of registers, a new management model and the purchase of a new tool were proposed (Service Tonic, developed by a company with the same name) to adequately manage incidents and service management.

Keywords: incident management / ITIL organism of the Peruvian state 


\section{Introducción}

El artículo se enfoca en la gestión de incidentes para las operaciones de soporte tecnológico, lo cual permite maximizar la eficacia de las tecnologías de información (TI) mediante procesos de cambio consolidados, integrados y automatizados. El nuevo modelo de gestión de incidencias permitirá lo siguiente:

- Restablecer el servicio acordado lo antes posible y con el mínimo impacto.

- Garantizar el más alto nivel de calidad y disponibilidad del servicio.

- Implementar los acuerdos de nivel de servicio (SLA) que aún no están definidos.

- Registrar en forma permanente los incidentes (trazabilidad).

- Identificar proactivamente las mejoras del servicio.

- Definir las bases para la generación de la gestión de la configuración de base de datos (CMDB, por sus siglas en inglés) de acuerdo a las buenas prácticas.

- Minimizar el riesgo de incidentes perdidos.

- Recolectar datos relacionados a la gestión.

\subsection{Diagnóstico situacional}

La Oficina Nacional de Gobierno Electrónico e Informática, en adelante ONGEl, órgano técnico especializado de la Presidencia del Consejo de Ministros (PCM), se encarga de "normar, coordinar, integrar y promover el desarrollo de la actividad informática en la Administración Pública" (DS N.º 066-2003-PCM), así como de "dirigir, implementar y supervisar la política nacional de gobierno electrónico e informática" (DS N. ${ }^{\circ} 067$ 2003-PCM). La ONGEl es el ente rector del Sistema Nacional de Informática, y brinda servicios de TI y gobierno electrónico a diversas entidades del estado; entendiéndose por gobierno electrónico al uso, por parte del estado, de las tecnologías de información y la comunicación (TIC) para brindar servicios e información a los ciudadanos.

\subsubsection{Política de gobierno electrónico}

De acuerdo al manual de gestión de la ONGEI (Yurivilca y Loayza, 2014), la política de gobierno electrónico se sustenta en diversos planes nacionales, tales como la Agenda Digital Peruana 2.0, Agenda de Competitividad 2012-2013, el Plan Bicentenario, la Estrategia Nacional de Gobierno Electrónico y la Política Nacional de Modernización de la Gestión Pública. 


\subsubsection{Clasificación de desarrollo en gobierno electrónico a nivel mundial}

La División de Administración Pública y Gestión del Desarrollo (DPADM) de las Naciones Unidas (2014) realiza una clasificación en materia de gobierno electrónico a partir de indicadores asociados a tres aspectos básicos:

i. Servicio en línea (Online Service Index, OSI)

ii. Infraestructura en telecomunicaciones (Telecommunication Infrastructure Index, TII)

iii. Capital humano (Human Capital Index, $\mathrm{HCl}$ ).

La Ongei, como ente rector del Sistema Nacional de Informática del Perú, es evaluada y clasificada según estos aspectos. En el ranking del 2014 (United Nations, 2014) se ubicó en el puesto 72, con un índice de 0,5435, clasificado como un nivel medio alto; ello demuestra un ascenso con respecto al 2012, en el cual se encontraba en el puesto 82 (United Nations, 2012). La Política Nacional de Gobierno Electrónico 2013-2017 tiene por finalidad alinearse a estos parámetros internacionales, y para esto debe mejorar la entrega de sus servicios de TI. E-Government Development Index.

\subsubsection{Diagnóstico situacional respecto al uso de TICs}

De acuerdo al análisis realizado en la ONGEI se identificó lo siguiente:

- Baja transparencia y demora en la atención a los ciudadanos.

- Inadecuado soporte de hardware.

- Falta de una estrategia coherente por parte del estado.

- Insuficientes mecanismos de coordinación.

- Falta de normas específicas en el uso de TI, alineadas a estándares internacionales.

- Baja disponibilidad de servicios públicos.

- Baja integración de los sistemas de gobierno.

- Falta de sensibilización y capacitación de funcionarios.

Adicionalmente, se identificó que la función de Mesa de Ayuda no estaba implementada adecuadamente. Los problemas que se detectaron fueron los siguientes: 
- No se disponía de un historial de incidencias de los servicios atendidos, publicados y puestos en marcha.

- No todos los servicios se encontraban amparados bajo la modalidad de acuerdo de nivel de servicios (SLA).

- No se disponía de un plan de mejora (SIP) respecto a la gestión de incidencias.

- No se disponía de un acuerdo de nivel de operación (OLA) respecto a la gestión de incidencias.

Lo detectado muestra una deficiente gestión en la atención de los incidentes y de procesos asociados que precisan ser mejorados. Si bien existe un modelo de gestión, este no se ajusta a las nuevas necesidades. En tal sentido, el artículo aborda la formulación de un nuevo modelo de gestión de incidentes, basado en ITIL V3.0, que contribuya a la inmediata restauración de servicios, manteniendo la continuidad de los servicios de los procesos de negocios en la ONGEl.

\subsection{Alcances y aportes}

\subsubsection{Alcances}

i. Se abordará la gestión de incidentes de la ONGEl a través de un modelo que se adecúe a las necesidades de la entidad.

ii. Se considerarán como usuarios de los servicios de TI a todas las entidades públicas del estado.

\subsubsection{Aportes}

Nivel interno

- Implementar un modelo de gestión de incidentes, aplicando ITIL V3.0 en el ente rector del Sistema Nacional de Informática.

- Garantizar la trazabilidad de información para los procesos de gestión de incidencias.

- Mejorar la calidad del servicio: menor tiempo de respuesta, disminución de reclamos y mayor nivel de satisfacción de los usuarios, entre otros.

- Brindar un inventario actualizado de activos y procedimientos del área de TI e Infraestructura Tecnológica en la ONGEI. 
Nivel externo

- Ofrecer un servicio idóneo a las entidades del estado.

- Brindar bases técnicas y funcionales para los servicios de Mesa de Ayuda.

- Ser modelo técnico y referencial para proyectos similares en el sector público.

\section{Metodología}

Con la finalidad de diseñar un modelo de gestión de incidentes basado en la metodología ITIL V3.0, que permita superar las deficiencias y la pérdida de calidad del servicio en la ONGEI, se procedió revisando y aplicando cada uno de los puntos que se detallan a continuación.

\subsection{Biblioteca de infraestructura de tecnología de información, ITIL}

De acuerdo al Office of Government Commerce (OGC, 2008), ITIL es el marco y fuente de buenas prácticas en la gestión del servicio, que permite establecer y mejorar sus capacidades. La norma ISO/IEC 20000 es la guía oficial y el estándar universal para las organizaciones, con el que lograrán capacidades de gestión auditadas y certificadas. ITIL ofrece un cuerpo de conocimientos útiles para alcanzar dicho estándar. Está integrado por los siguientes componentes:

- El núcleo de ITIL: Guía de mejores prácticas aplicables a todos los tipos de organizaciones que prestan servicios a una empresa.

- Publicaciones complementarias ITIL: Conjunto de publicaciones con guía específica para sectores de la industria, tipos de organización, modelos operativos y arquitecturas tecnológicas.

El núcleo de ITIL está constituido por cinco publicaciones:

- Estrategia del servicio

- $\quad$ Diseño del servicio

- Transición del servicio

- Operación del servicio

- Mejora continua del servicio (CSI) 
Figura 1. Fases de ITIL V3

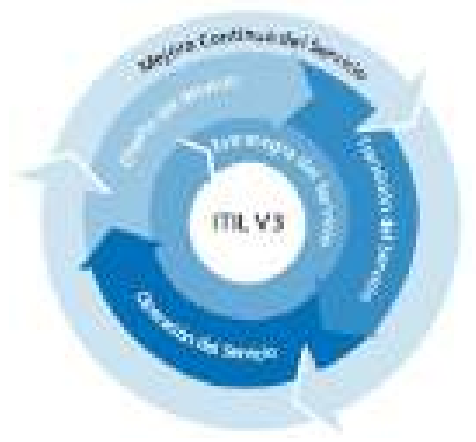

Fuente: Bon et al. (2008, p. 22)

Figura 2. Procesos y funciones considerados en ITIL V3

\section{ITIL Edition 2011 Components}
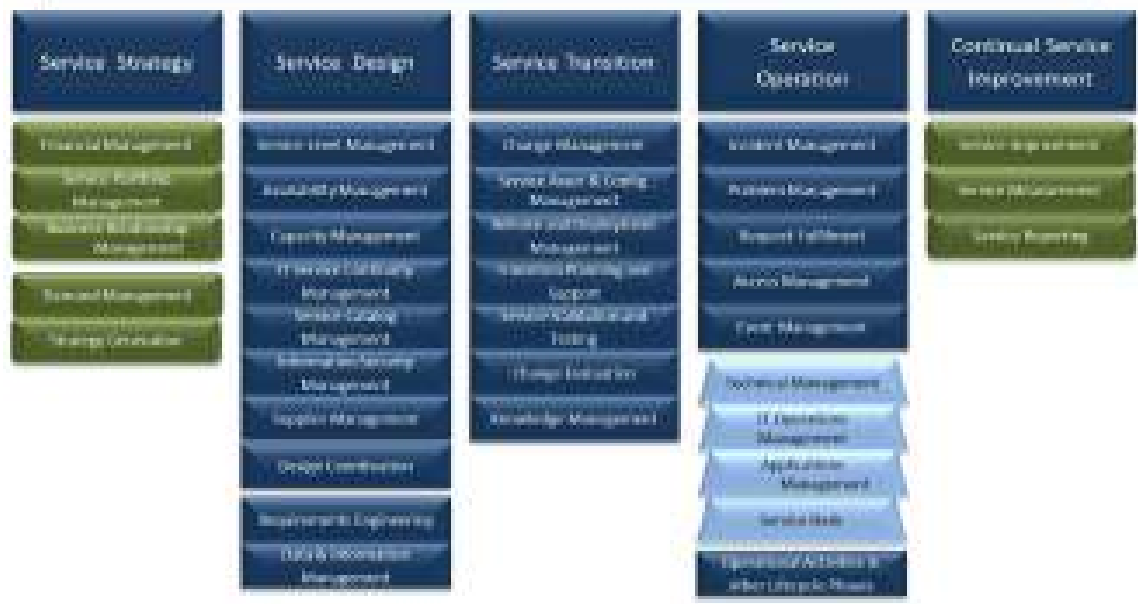

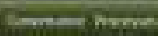
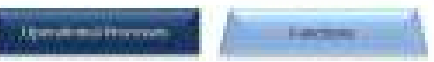

Fuente: Bon et al. (2008, p. 22)

\subsection{Gestión de incidencias}

La gestión de incidencias tiene como objetivo resolver, de manera rápida y eficaz, cualquier problema que cause una interrupción en el servicio. El proceso ITIL V3 gestión de incidentes contempla los siguientes subprocesos: 
i. Soporte a gestión de incidentes

ii. Registro y categorización de incidentes

iii. Resolución de incidentes por el soporte de primera línea

iv. Resolución de incidentes por el soporte de segunda línea

v. Gestión de incidentes graves

vi. Monitorización y escalado de incidentes

vii. Cierre y evaluación de incidentes

viii. Información proactiva a usuarios

ix. Informes de gestión de incidentes

Figura 3. Proceso de la gestión de incidentes

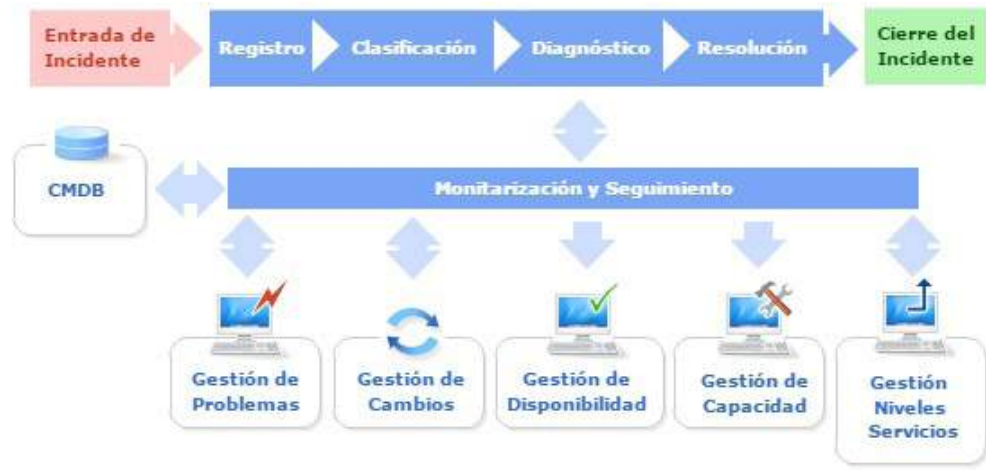

Fuente: Osiatis S.A. (s.f.)

Los objetivos principales de la gestión de incidencias son:

i. Detección y registro del incidente

ii. Clasificación y soporte inicial

iii. Investigación y diagnóstico

iv. Solución y restablecimiento del servicio

v. Cierre del incidente

vi. Monitorización, seguimiento y comunicación del incidente 
El proceso de gestión de incidentes tiene una estrecha relación con la función Mesa de Ayuda, siendo una parte fundamental para una correcta entrega de servicios de Tl.

\section{Análisis del sistema de gestión de ONGEI}

\subsection{Sistema de gestión de ONGEI}

El sistema de gestión ofrece un enfoque metodológico para dirigir y controlar la organización de acuerdo al cumplimiento de los objetivos de gestión y la mejora de su desempeño. Su implementación toma como referencia el modelo de sistema de gestión de calidad ISO 9001.

\section{Modelo de gestión}

Permite implementar y mejorar el sistema de gestión para el cumplimiento integral de los requisitos establecidos en las normas y políticas vigentes que, en materia de modernización, ha promovido el gobierno. Este modelo, como se indica en la figura adjunta, comprende los siguientes elementos:

\section{Figura 4. Modelo de gestión de la ONGEI}

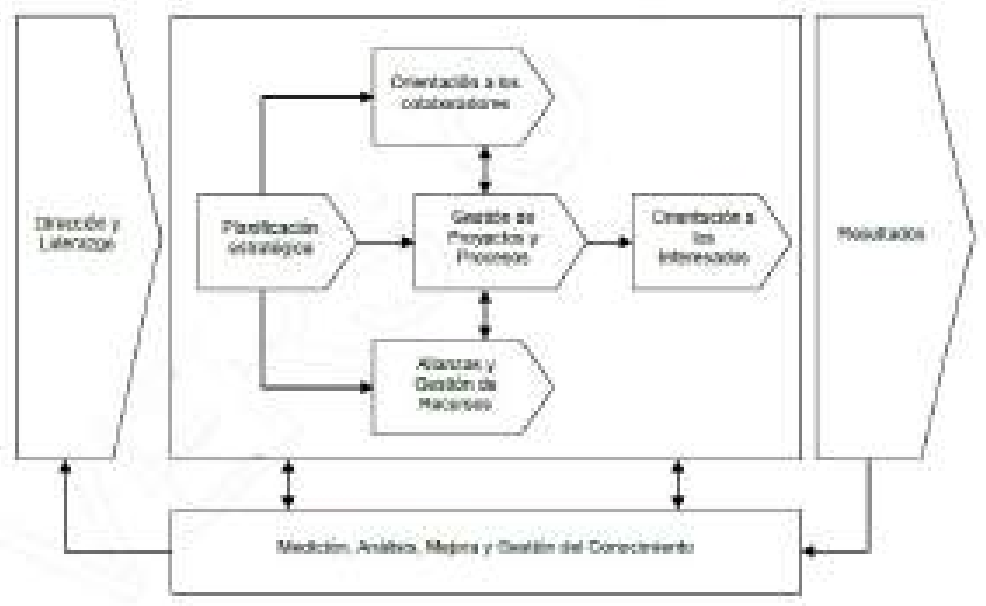

Fuente: Yurivilca y Loayza (2014, p. 20)

Documentación del sistema de gestión

Los documentos del sistema de gestión serán controlados de acuerdo al procedimiento SGO-PRO-001 elaboración y control de documentos, que comprende la elaboración, revisión, aprobación, distribución, mantenimiento y uso de los documentos del sistema de gestión de ONGEl. 


\subsection{Servicios de TI entregados por la ONGEI}

Los servicios proporcionados por la ONGEl son los siguientes:

- $\quad$ Plataforma de Interoperabilidad del estado (PIDE)

- $\quad$ Sayhuite

- Infraestructura de Datos Espaciales del Perú (IDEP)

- Yachaywasi Digital

- Regulación técnico-normativa sobre gobierno electrónico

- Portales del estado

- Coordinadora de Emergencias de Redes Teleinformáticas de Perú (PeCERT)

- Asesoría legal

- Capacitación de funcionarios del SIN y funcionarios No TI

Varios de estos servicios utilizan servicios de la plataforma PIDE para poder generar nuevos servicios.

Figura 5. Esquema de funcionamiento de la PIDE

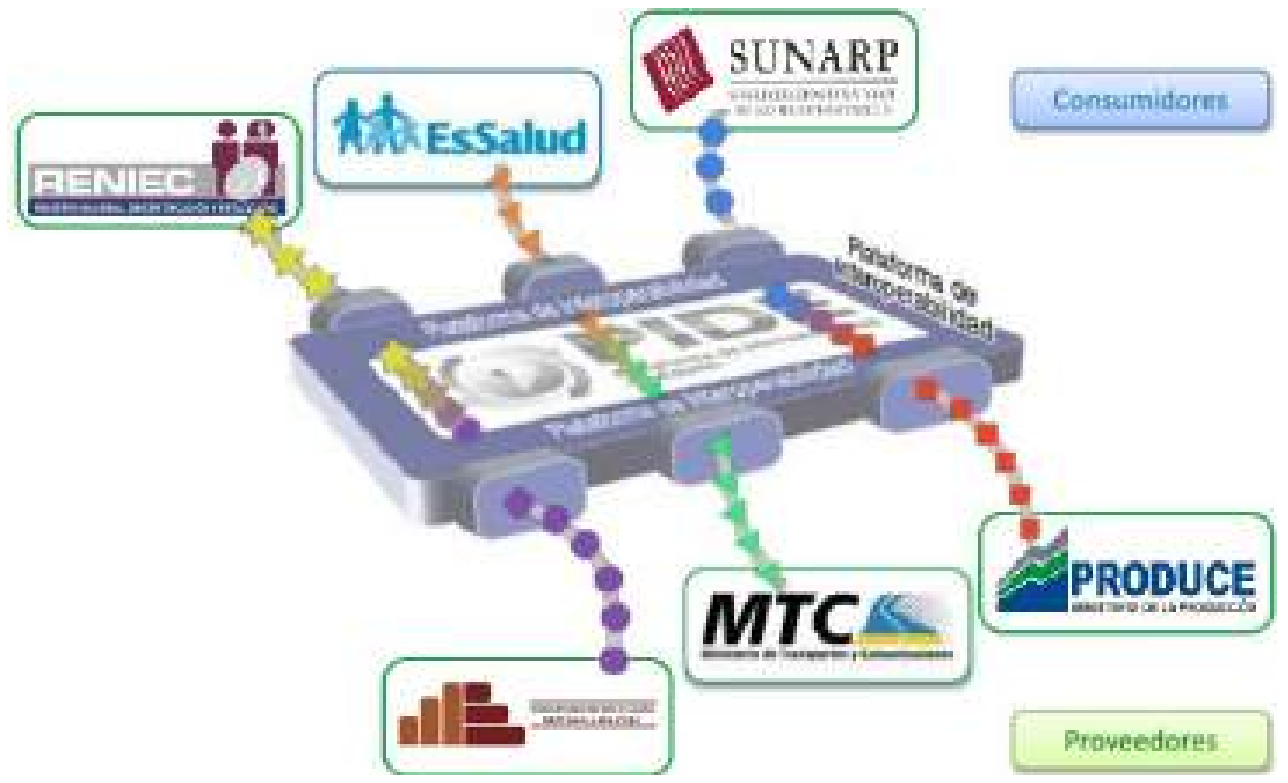

Fuente: ONGEI (2012) 
Mapa de procesos

El modelo de operación por procesos de la ONGEI se muestra en la figura 6.

Figura 6. Mapa de procesos de la ONGEI

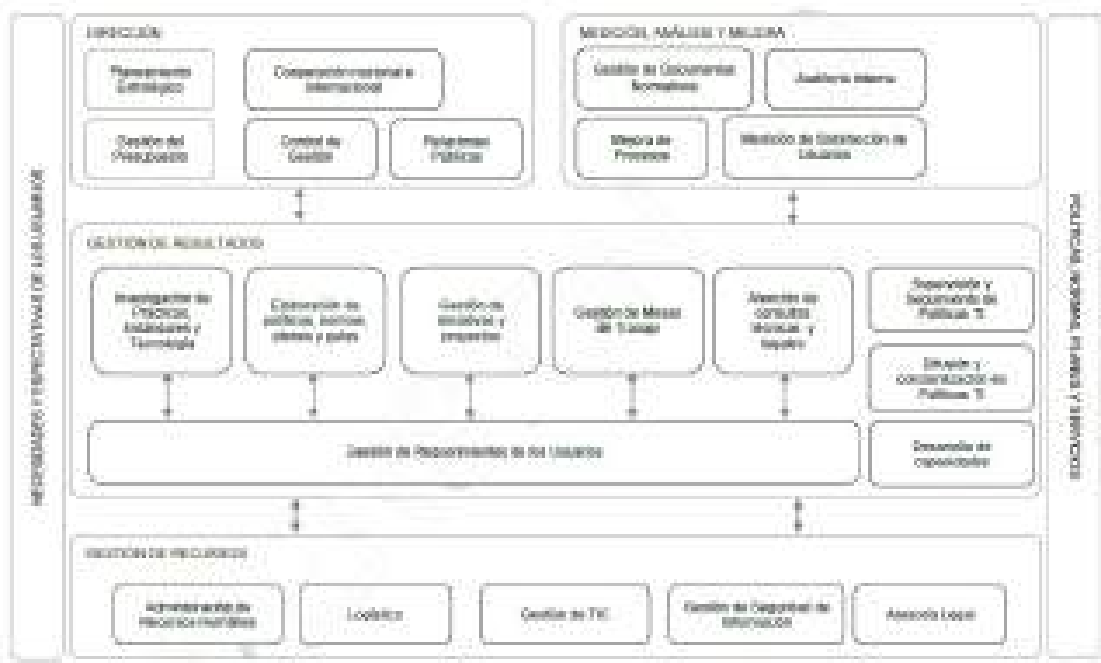

Fuente: Yurivilca y Loayza (2014, p. 25)

\subsection{Gestión de incidentes actual en la ONGEI}

\subsubsection{Clientes ONGEI}

En la figura 7 se muestra la evolución de los servicios de TI y cómo los diferentes usuarios se han ido clasificando de acuerdo al nivel de complejidad de los servicios que utilizan.

Figura 7. Evolución y usuarios de servicios de TI
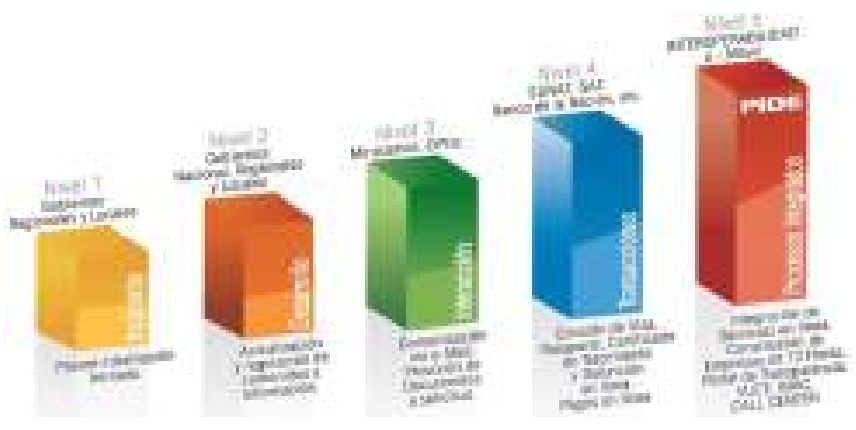

Fuente: Yurivilca y Loayza (2014) 


\subsubsection{Servicios técnicos de la ONGEI}

La figura 8 muestra cómo interactúan los servicios técnicos con los componentes de $\mathrm{TI}$, acuerdos de $\mathrm{Tl}$ y soporte $\mathrm{TI}$.

Figura 8. Interacción de servicios técnicos y activos de servicios

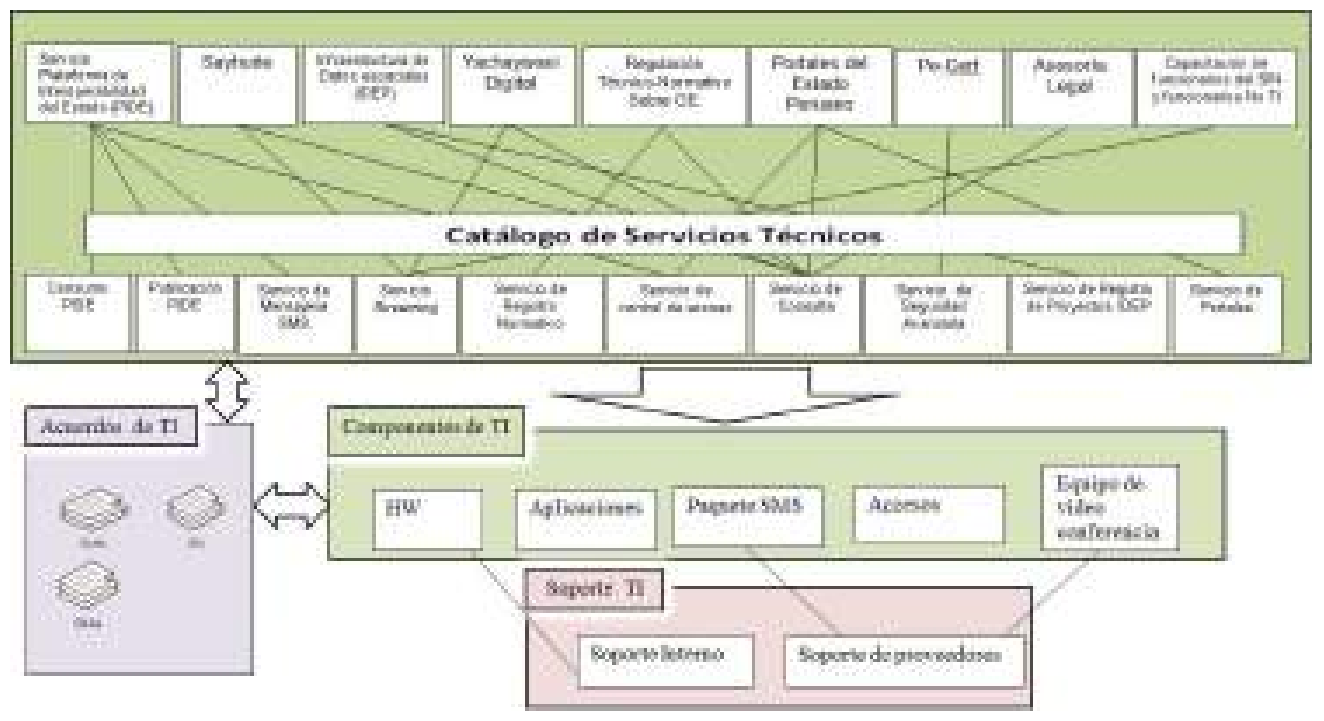

Fuente: Yurivilca y Loayza (2014)

\subsubsection{Estado situacional de la atención de incidentes}

La atención de incidentes no disponía de un proceso estandarizado, lo que originaba irregularidades en su ingreso, principalmente debido a los siguientes motivos:

- El incidente no siempre ingresaba a donde debería ser el primer punto de contacto entre la entidad gubernamental y la ONGEI. Se detectaron casos que son atendidos directamente por el equipo de soporte, inclusive sin ser registrados.

- A menudo el incidente ingresa por trámite documentario, lo que genera retrasos en la atención, así como la consecuente insatisfacción en los usuarios.

- Los roles y responsabilidades no se encuentran adecuadamente definidos, de manera que los equipos de trabajo realizaban tareas que no les correspondían. 
- Duplicidad en el registro de incidentes, por lo que se generaban conflictos con el usuario y ello repercutía en la imagen de la organización.

Ambiente tecnológico

Los incidentes eran registrados en un archivo Excel en sistema bitácora, porque el sistema anterior no permitía gestionar los nuevos incidentes para los nuevos servicios creados. A este archivo tenían acceso los tres equipos de soporte: Mesa de Ayuda, soporte Tl y seguridad de la información. Este sistema se sigue utilizando para el registro de inventario de la infraestructura de TI y documentación de problemas resueltos.

\section{Gestión de incidentes}

Tiene por objetivo restablecer lo más rápido posible el servicio normal de la operación de los portales web, servicios SOA e infraestructura tecnológica. Como consecuencia del aumento de servicios más sofisticados, se presentaban deficiencias en la atención de incidentes; en algunos casos estos no son atendidos ni registrados por la Mesa de Ayuda.

En la gestión de incidencias, ver figura 9, no existía el manejo de severidades, de los tiempos de respuesta y los tiempos de restauración; por lo que no se podía identificar a los incidentes registrados resueltos dentro de los parámetros normales. Por tal motivo, no se realizaba un estricto control en la gestión de los incidentes.

\section{Figura 9. Flujo de proceso de la gestión de incidentes}

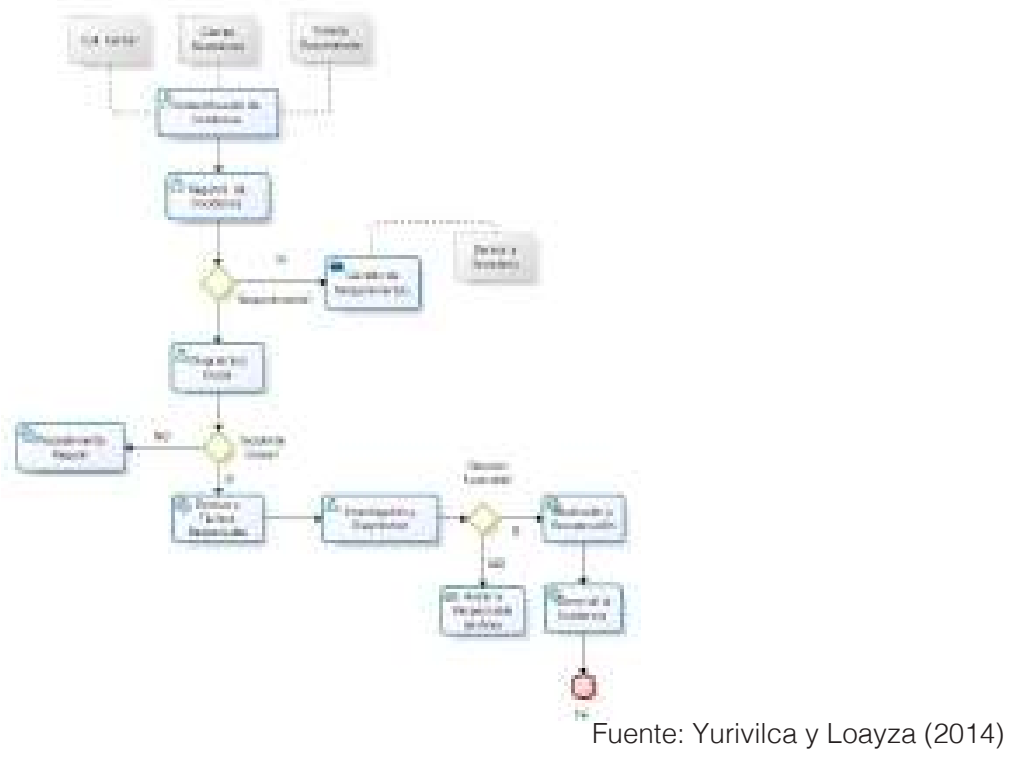


En la figura 10 se muestra el modelo de madurez de la gestión de servicios.

\section{Figura 10. Servicios de TI. Modelo de madurez de gestión}

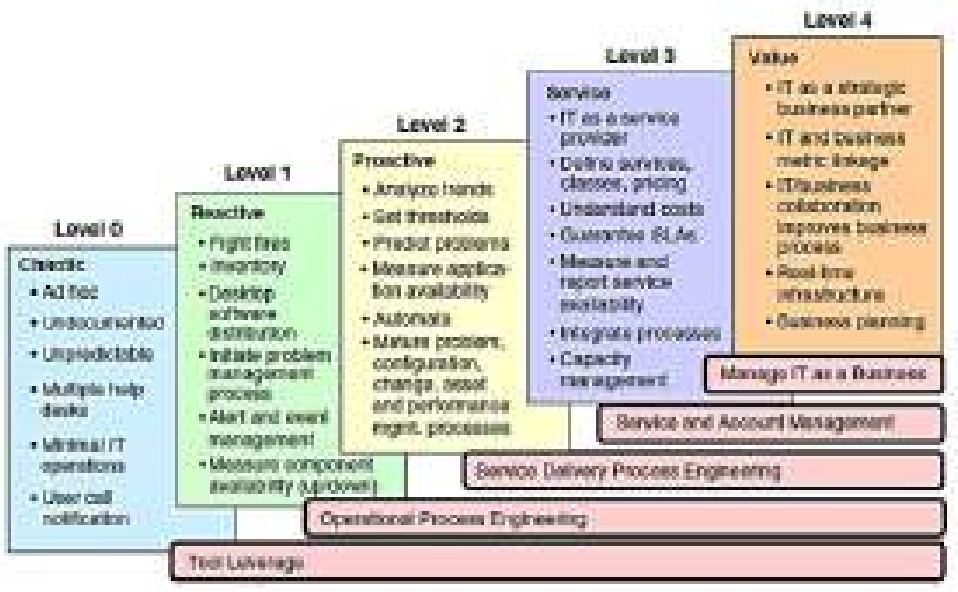

Fuente: Tang y Todo (2013, p. 2)

En la tabla 1 se presenta el análisis realizado donde se observa que la ONGEI se encuentra en el nivel 0 de madurez.

\section{Tabla 1. Cuadro comparativo}

\begin{tabular}{|c|c|c|c|}
\hline \multirow{2}{*}{ Elementos ITIL } & \multicolumn{2}{|c|}{ ONGEI } & \multirow{2}{*}{ Observación } \\
\hline & Sí & No & \\
\hline Estructura organizacional ITIL & $x$ & & $\begin{array}{l}\text { Falta de madurez en la organización y redefinir } \\
\text { procesos. }\end{array}$ \\
\hline Catálogo de servicios & $x$ & & $\begin{array}{l}\text { No se encuentra actualizado. Es necesario } \\
\text { modificar o crear otro modelo. }\end{array}$ \\
\hline $\begin{array}{l}\text { Acuerdos de nivel de } \\
\text { servicios (SLA) }\end{array}$ & $x$ & & No se encuentra actualizado. Falta redefinir. \\
\hline $\begin{array}{l}\text { Acuerdos de nivel de } \\
\text { operación (OLA) }\end{array}$ & & $x$ & $\begin{array}{l}\text { No se encuentra definido. Se necesita establecer } \\
\text { acuerdos internos. }\end{array}$ \\
\hline Gestión de incidentes & $x$ & & $\begin{array}{l}\text { No existe una adecuada administración de } \\
\text { incidentes. Necesita implementar } \\
\text { un nuevo modelo. }\end{array}$ \\
\hline
\end{tabular}




\subsection{Estadística de gestión de incidentes en la ONGEI}

Dado que no se disponía de un formato adecuado de registro, no se midieron los tiempos y estados de los incidentes. Con el apoyo del personal de Mesa de Ayuda se obtuvieron las estadísticas (figura 11), relacionadas al estado de incidentes del último trimestre del 2014.

Figura 11. Incidentes por estado y mes

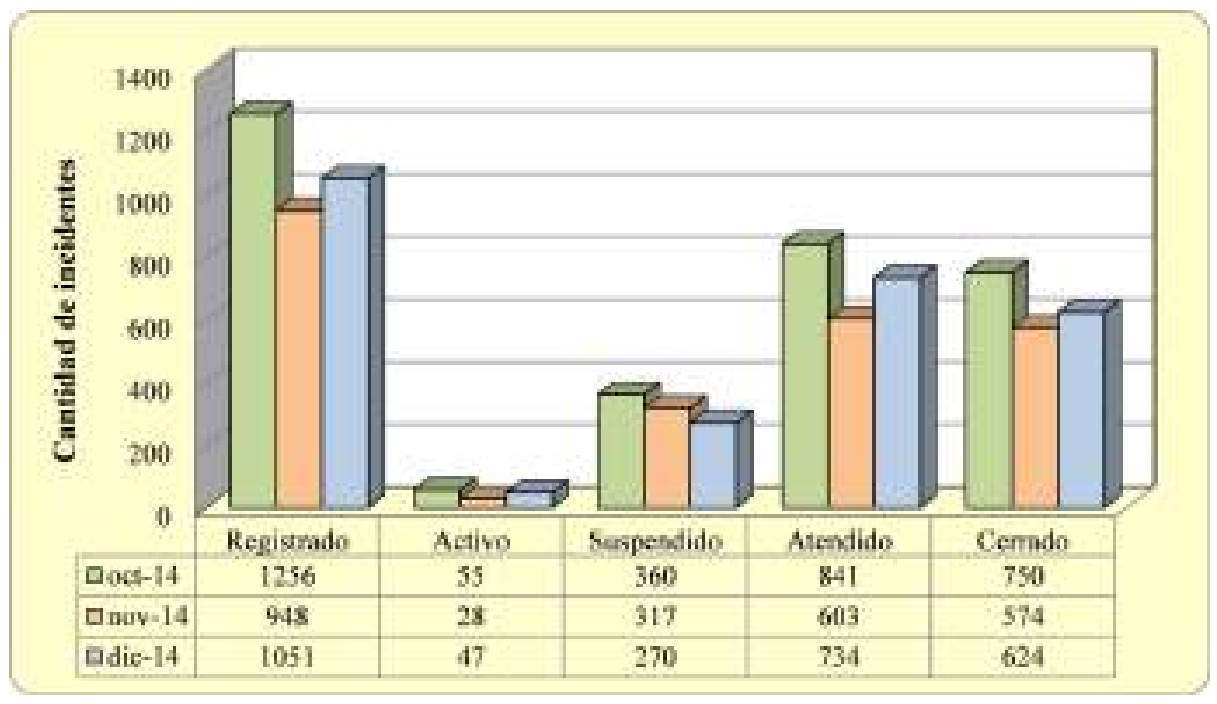

Fuente: ONGEI. Equipo de Mesa de Ayuda

- Registrado: Estado de un incidente al momento de creación y registro.

- Activo: Incidente derivado al portal de servicio y que está siendo resuelto.

- Suspendido: Incidente registrado, derivado o no, que ha sido suspendido.

- Atendido: Incidente registrado y que ya está resuelto, espera conformidad de usuario.

- Cerrado: Incidente atendido y que el usuario ya dio su conformidad y ha sido verificado.

3.5. Conclusiones de la situación actual

i. No hay una política definida de registro y atención de incidentes.

ii. El registro actual es una limitante para obtener indicadores más puntuales. 
iii. Existe un alto de nivel de incumplimiento por parte de las entidades del Estado en temas relacionados a POI, Enriap y algunos otros servicios obligatorios, debido a vacíos en los SLA (niveles de acuerdo de servicio).

iv. Se necesita establecer procedimientos claros y que cuenten con la requerida continuidad, independientemente de los cambios administrativos de los gobiernos de turno.

v. El modelo actual no soporta eficientemente los incidentes generados para los nuevos servicios que actualmente entrega la ONGEl.

vi. El registro de incidentes lo realizan los diversos equipos de trabajo, lo que ocasiona duplicidad de esfuerzos y poca certeza en el registro de información.

vii. No permite determinar si los incidentes registrados fueron de alta o baja incidencia, cuántos de estos fueron recurrentes y se convirtieron en problemas.

\section{Propuesta de solución}

El modelo propuesto está soportado en las buenas prácticas de ITIL aplicado a la gestión de incidentes. Durante la implementación hubo cambios en la Presidencia de Consejo de Ministros (PCM), por lo cual se involucró a nuevas personas para su puesta en marcha; estos inconvenientes se superaron satisfactoriamente gracias a la participación del nuevo personal. En una primera fase el modelo se aplicará como una prueba piloto en la organización y así poder evaluar el impacto organizacional; posteriormente se tendrá una fase de reorganización.

\subsection{Estrategia del servicio del modelo propuesto}

Esta fase el modelo se ajusta a la visión estratégica y política de la ONGEl.

\subsubsection{Objetivos}

i. Proporcionar un modelo estructurado para la recepción, clasificación y registro de incidentes enviados por las entidades gubernamentales, así como su gestión eficiente con una mayor calidad de atención.

ii. Generar automáticamente notificaciones vía correo electrónico dirigidas al usuario, al momento de que este registre un incidente.

iii. Crear las condiciones para una adecuada trazabilidad de los incidentes, al igual que mantener informados a los usuarios sobre el estado de sus requerimientos. 
iv. Reducir el nivel de incumplimiento de los acuerdos de nivel de servicios (SLA).

v. Establecer una línea base de indicadores para la madurez del modelo propuesto.

vi. Proponer un modelo de acuerdo de nivel operativo (OLA) para un mejor control de los tiempos de respuesta en los servicios involucrados.

\subsubsection{Estrategias a implementar}

i. Ser el único punto de contacto en relación a la gestión de incidentes.

ii. Diseñar un modelo de gestión de incidentes acorde a la organización y a los objetivos planteados.

iii. Contar con una base de datos de conocimientos, con el fin de brindar una atención rápida a los usuarios dentro del soporte de primera línea.

iv. Crear un nuevo de catálogo de servicios que delimite las funciones y compromisos de la Mesa de Ayuda, el cual se adapte a las necesidades de los usuarios de la ONGEI.

v. Diseñar un plan de capacitación en ITIL V3 para los involucrados, que facilite la adecuación a los cambios propuestos. Igualmente para el personal de Mesa de Ayuda con respecto al manejo de situaciones de atención a los usuarios.

vi. Proponer una nueva organización para la gestión de TI, que permita un mejor control de los incidentes. Para ello se implementará el área de control, liderada por el gestor de servicios de TI. El cambio se basa en el siguiente esquema: 
Figura 12. Nuevo organigrama para el área de TI

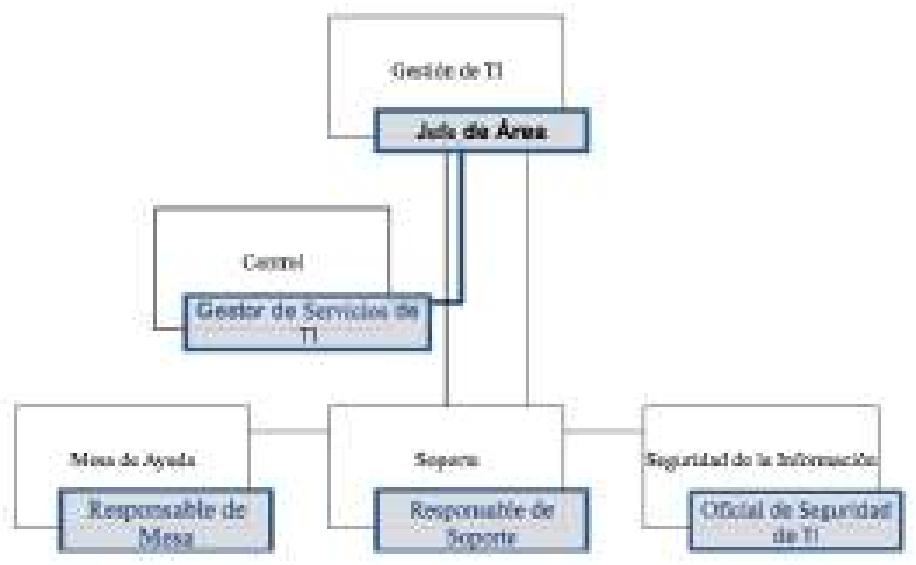

Elaboración propia

\subsection{Diseño del servicio del modelo propuesto}

4.2.1. Identificar actores para la asignación de roles

A continuación se definen los roles propuestos para la ONGEl.

Tabla 2. Matriz RACI para la gestión de incidentes en la ONGEI

\begin{tabular}{|c|c|c|c|c|c|c|}
\hline \multirow[b]{2}{*}{ Actividades } & \multicolumn{6}{|c|}{ Roles } \\
\hline & $\begin{array}{l}\text { Usuario } \\
\text { de TI }\end{array}$ & $\begin{array}{l}\text { Soporte de } \\
\text { primer nivel } \\
\text { HelpDesk }\end{array}$ & $\begin{array}{l}\text { Soporte } \\
\text { técnico de } \\
\text { nivel N }\end{array}$ & $\begin{array}{l}\text { Responsable } \\
\text { de área }\end{array}$ & $\begin{array}{l}\text { Gestor de } \\
\text { incidentes }\end{array}$ & $\begin{array}{c}\text { Gestor de } \\
\text { servicios } \\
\text { de TI }\end{array}$ \\
\hline Identificación & C & $\mathrm{R}$ & & & $A$ & I/C \\
\hline $\begin{array}{l}\text { Registro, } \\
\text { clasificación y } \\
\text { soporte inicial }\end{array}$ & C & $\mathrm{R}$ & I & & A & $\mathrm{I} / \mathrm{C}$ \\
\hline $\begin{array}{l}\text { Investigación y } \\
\text { diagnóstico }\end{array}$ & C & $\mathrm{I} / \mathrm{R}$ & $\mathrm{R}$ & 1 & A & $\mathrm{I} / \mathrm{C}$ \\
\hline $\begin{array}{l}\text { Solución y } \\
\text { recuperación }\end{array}$ & I & I/R & $\mathrm{R}$ & & A & $\mathrm{I} / \mathrm{C}$ \\
\hline Cierre & $\mathrm{C}$ & $\mathrm{R}$ & & & $A$ & $\mathrm{I} / \mathrm{C}$ \\
\hline
\end{tabular}

Elaboración propia 


\subsubsection{Indicadores iniciales del modelo}

Se definieron los siguientes indicadores para medir el nivel de madurez en la implementación del modelo y obtener una línea base que permitirá realizar los ajustes necesarios:

- $\quad$ Tiempo mínimo y máximo de atención de un incidente en un mes

- Porcentaje de reducción de incidentes

- Número total de incidentes comunes

- Total de incidentes agrupados por tipo de prioridad

- Incidentes derivados a equipos de trabajo clasificados por tipo de prioridad

- Cantidad de incidentes agrupados por categoría

- Número incidentes que desembocaron en problemas

\subsubsection{Categorización de incidentes}

En la tabla 3 se muestra una categorización, por niveles, para cada una de las categorías de los incidentes registrados.

\section{Tabla 3. Categorización de incidentes}

\begin{tabular}{|l|c|c|}
\multicolumn{1}{|c|}{ Categoría } & Nivel 1 & Nivel 2 \\
\hline Accesos & $\times$ & \\
\hline Consultas & $\times$ & \\
\hline Hardware & & $\times$ \\
\hline Software & & $\times$ \\
\hline Comunicaciones & & $\times$ \\
\hline Equipos & $x$ & \\
\hline Aplicaciones & & \\
\hline
\end{tabular}

Nota: Si el incidente requiere la participación de especialistas, entonces, se otorgará el nivel 3.

Elaboración propia basado en registros de la ONGEI. 


\subsubsection{Niveles de escalamiento por niveles de impacto}

En la tabla 4 se brinda una descripción de los niveles de impacto que se propusieron:

\section{Tabla 4. Descripción de los niveles impacto propuesto}

\begin{tabular}{|c|c|}
\hline Impacto & Descripción \\
\hline Crítico & $\begin{array}{l}\text { Alto impacto en las funciones críticas del negocio, por indisponibilidad o degradación } \\
\text { excesiva de desempeño de las aplicaciones o servicios. Requiere solución inmediata. } \\
\text { No existe workaround. }\end{array}$ \\
\hline 2 & $\begin{array}{l}\text { Impacto significativo en alguna de las funciones del negocio, por indisponibilidad o } \\
\text { degradación de desempeño en las aplicaciones o servicios. El evento está controlado } \\
\text { dado que existe un workaround y no requiere de una solución inmediata, los usuarios } \\
\text { pueden esperar la restauración del servicio. La solución definitiva debe estar programada. }\end{array}$ \\
\hline Medio & $\begin{array}{l}\text { Impacto moderado en alguna de las funciones del negocio, por degradación leve de } \\
\text { desempeño de las aplicaciones o servicios. El evento implica un número reducido de } \\
\text { usuarios o clientes afectados y es de poca visibilidad. Existe un plan alternativo de } \\
\text { solución y se puede esperar la solución definitiva. }\end{array}$ \\
\hline Bajo & $\begin{array}{l}\text { Bajo impacto en las funciones no críticas del negocio, el usuario puede esperar por } \\
\text { una fecha determinada para la solución definitiva. Puede continuar con sus tareas } \\
\text { críticas de operación, se mantiene la funcionalidad y el desempeño de las aplicaciones } \\
\text { o servicios. }\end{array}$ \\
\hline
\end{tabular}

Elaboración propia

\section{Tabla 5. Definición de tiempos de respuesta}

\begin{tabular}{|c|c|c|}
\hline Impacto & Tiempo de respuesta & Tiempo de restauración \\
\hline Critico & $\begin{array}{l}70 \% \text { de incidentes dentro } \\
\text { de } 15 \text { minutos }\end{array}$ & $\begin{array}{l}95 \% \text { de incidentes dentro de } 4 \text { horas } \\
100 \% \text { de incidentes dentro de } 6 \text { horas }\end{array}$ \\
\hline Alto & $\begin{array}{l}70 \% \text { de incidentes dentro } \\
\text { de } 30 \text { minutos }\end{array}$ & $\begin{array}{l}95 \% \text { de incidentes dentro de } 8 \text { horas } \\
100 \% \text { de incidentes dentro de } 12 \text { horas }\end{array}$ \\
\hline Medio & $\begin{array}{l}70 \% \text { de incidentes dentro } \\
\text { de } 8 \text { horas }\end{array}$ & 95 \% de incidentes dentro de 3 días hábiles \\
\hline Bajo & $\begin{array}{l}70 \text { \% de incidentes dentro } \\
\text { de } 1 \text { día hábil }\end{array}$ & 95 \% de incidentes dentro de 5 días hábiles \\
\hline
\end{tabular}

Elaboración propia 


\subsubsection{Criterios de cambio entre niveles de impacto}

Impacto - Downgrade:

De 1 a 2: (a) no afecta aplicaciones críticas del negocio o el impacto es significativo en alguna de las funciones del negocio, (b) existe workaround o se puede esperar a la solución, y (c) el desempeño de la aplicación permite trabajar de manera limitada.

De 2 a 3: El impacto es moderado y se reduce solo para algunas funciones del negocio.

De 3 a 4: El impacto es bajo en funciones no críticas del negocio.

Impacto - Upgrade:

De 4 a 3: El incidente impacta moderadamente a un grupo de usuarios o impacta alguna función del negocio.

De 3 a 2: (a) el incidente impacta significativamente a vanas funciones de negocio y (b) el incidente tiene visibilidad sobre clientes externos.

De 2 a 1: (a) no existe workaround o (b) impacto alto en funciones críticas del negocio.

\subsubsection{Modelo Propuesto}

Monitoreo de servicios de TI

El equipo de Soporte de TI realizará actividades de monitoreo e informará al equipo de Mesa de Ayuda. Algunos de los procedimientos propuestos son los siguientes:

i. Verificación de servidores físicos y virtuales de la PIDE y otros servicios

ii. Realización de pruebas de mensajería SMS

iii. Verificación diaria de usuarios bloqueados en el directorio activo

Los siguientes flujos de proceso fueron elaborados con el uso del software Bizagi Process Modeler. 
Figura 13. Flujo de proceso para la verificación diaria de servidores

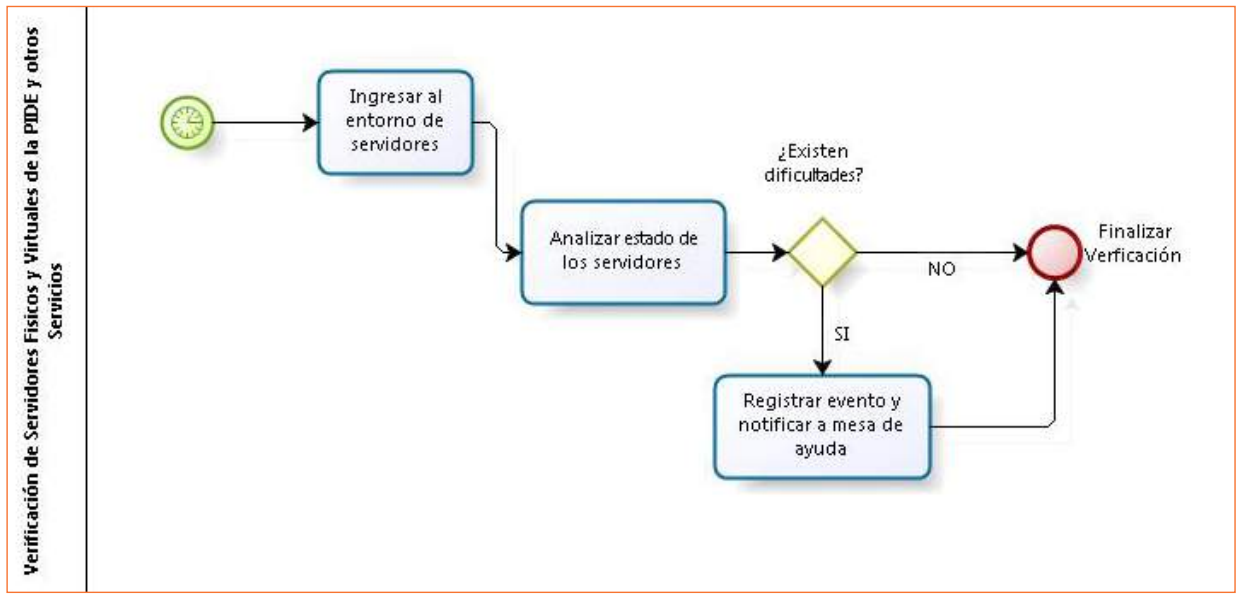

Elaboración propia

Figura 14. Flujo de proceso para pruebas diarias de servicios SMS

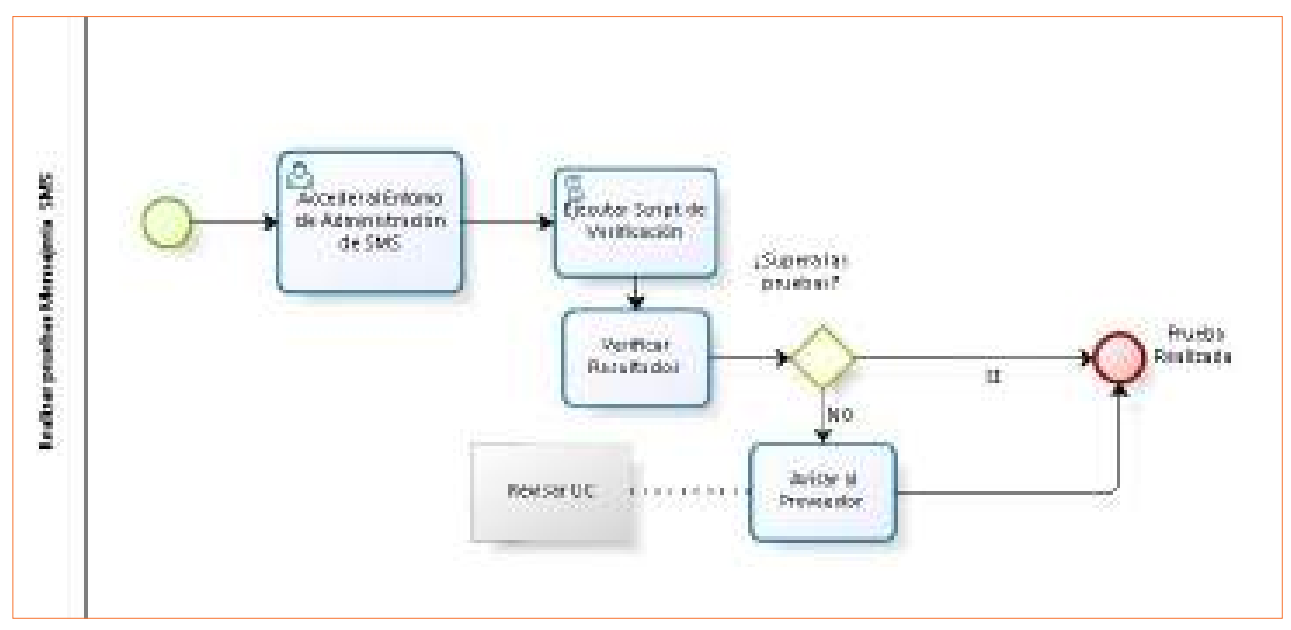

Elaboración propia 
Figura 15. Flujo de proceso para las pruebas la verificación de usuarios bloqueados

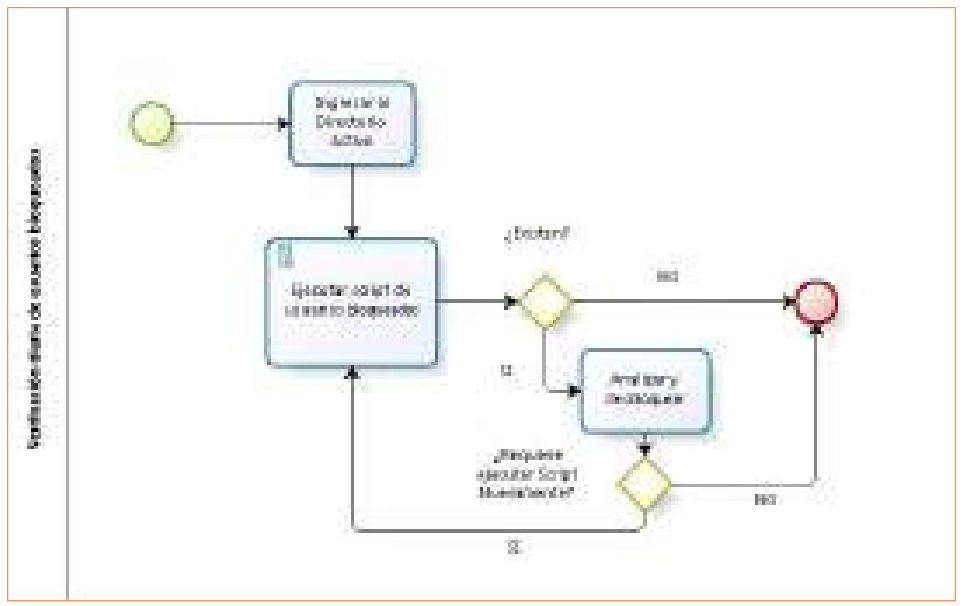

Elaboración propia

El modelo propuesto busca incentivar las acciones preventivas que permitan analizar las tendencias presentadas por alertas y eventos. Para cubrir la ausencia de algunos procesos necesarios se habilitará un módulo gestionado por el equipo de soporte, dentro de la herramienta ServiceTonic, exclusiva para el registro de eventos. 


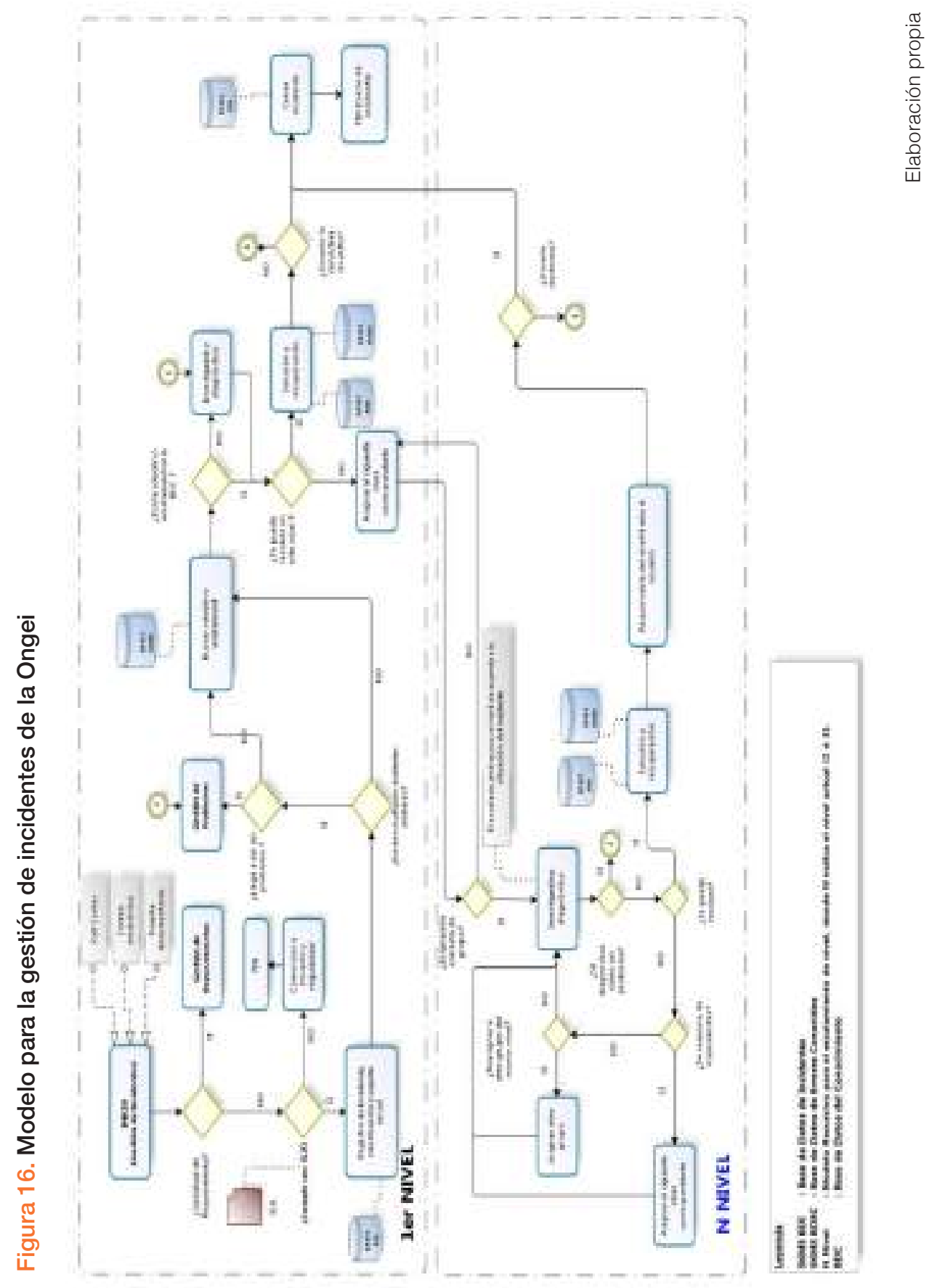


Descripción del proceso

i. Identificación

El modelo presenta tres formas de notificar un incidente: trámite documentario, call center y correo electrónico. Los incidentes que llegan a través de trámite documentario estarán contemplados dentro del nuevo SLA; luego se verifica si es una solicitud de requerimiento y de ser así se deriva a los responsables de la gestión de requerimientos, caso contrario, se continúa con el flujo; el siguiente paso es verificar si cumple los niveles de acuerdo de servicio, si fuese el caso se pasa a la siguiente actividad de registro; caso contrario, se le comunica al usuario para que regularice el ingreso del incidente de acuerdo al SLA.

\section{ii. Registro, clasificación y soporte inicial del incidente}

Los incidentes son registrados de acuerdo al siguiente formato: tipo de incidente, fecha, origen de notificación, entidad solicitante, nombre del usuario, prioridad, "asignado a", estado, categoría y subcategoría; código de identificación, descripción, números telefónicos y correo electrónico del usuario, indicador relacionado con otro incidente, registro de cambio de impacto e indicador de registro por excepción del SLA. Dentro de la priorización de los incidentes se considera lo siguiente:

- Impacto: Importancia del incidente según cómo afecta a los procesos de negocio.

- Urgencia: Según los tiempos señalados en el acuerdo de nivel de servicios

\section{Figura 17. Matriz impacto-urgencia}

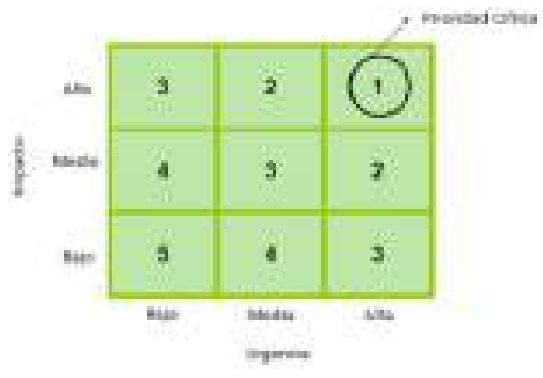

Fuente: Office of Government Commerce (2007, p. 51)

Es necesario tener presente que la prioridad del incidente puede cambiar durante su ciclo de vida. A partir del registro se procederá a verificar si el incidente presenta casos similares, si no hubiera coincidencia se procederá a buscar una solución o workaround en la Base de Datos del Conocimiento (BDC); caso contrario 
se verificaría si el incidente llega a ser un problema (impacto critico); si es así, se deriva al proceso de gestión de problemas que se encargará de determinar detalladamente las causas que lo originan; de otra manera continuaría con la búsqueda de la solución en la BDC; si existe la solución, se procede a verificar si puede ser resuelto en el presente nivel, si es posible, se pasa a la actividad de solución y recuperación, de lo contrario se hará el escalamiento respectivo. Si es requerido un escalamiento jerárquico, se hará a partir del segundo nivel.

iii. Investigación y diagnóstico

A partir de la información recogida del soporte inicial, se buscará reponer el servicio, dependiendo su grado de severidad; si no puede ser resuelto en el primer nivel, se procederá a buscar una solución en el siguiente nivel técnico de soporte, considerando a los grupos del mismo nivel involucrados en el incidente. En caso no pueda ser resuelto en el segundo nivel, se procederá a escalar al tercer nivel, donde estarían considerados los expertos y proveedores de servicios.

iv. Solución, recuperación y cierre del incidente

Cuando se haya solucionado el incidente, se procederá a:

- Confirmar con los usuarios la solución satisfactoria del incidente.

- Incorporar el proceso de resolución al sistema de gestión de conocimiento.

- Reclasificar el incidente si fuera necesario.

- Actualizar la información en base de datos de gestión de configuraciones (CMDB) sobre los elementos de configuración (Cl) implicados en el incidente.

- Medir el grado de satisfacción del usuario. Si no es satisfactoria la respuesta, se procederá de nuevo con la actividad de investigación y diagnóstico.

\subsubsection{Relación del modelo propuesto con otros procesos y funciones}

En el modelo propuesto se considera necesario brindar las pautas para mejorar la Mesa de Ayuda, para lo cual se propone implementar algunos procesos de ITIL. 
Figura 18. Impacto del modelo propuesto con la función de Mesa de Ayuda

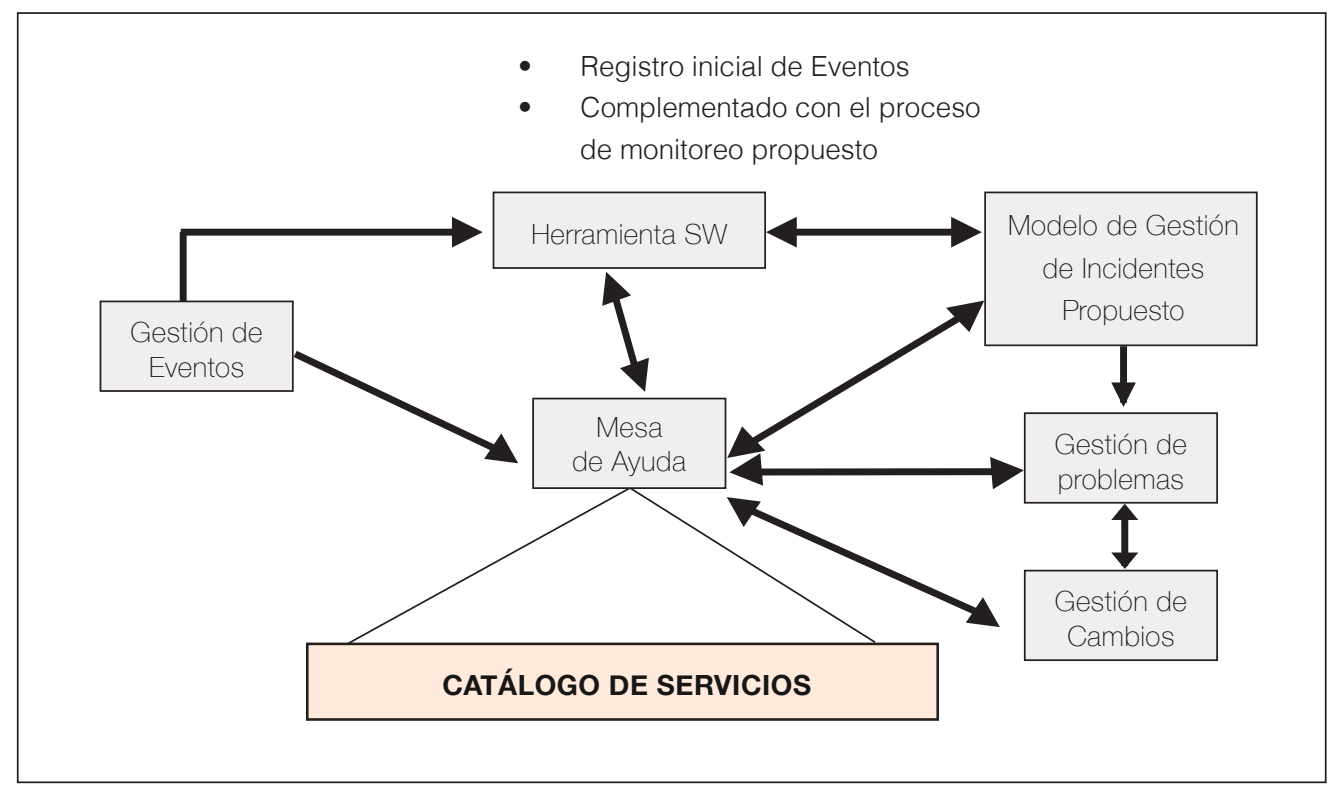

Elaboración propia

Se observa que la función de Mesa de Ayuda se encuentra respaldada por un modelo de gestión de incidentes mejorado. En las líneas discontinuas se ha señalado a la gestión de eventos como un proceso no implementado, pero que es importante para la detección temprana de incidentes. Para esto, dentro de la herramienta se activó un módulo básico para su registro; esto se complementa con los procesos de monitoreo propuestos.

La gestión de problemas precisa de ajustes y mejoras que no se encuentran contemplados en el presente artículo; dentro del esquema se observa que la gestión de cambios es atendida por el mismo grupo de personas de gestión de problemas y que todo cambio será supervisado por la Mesa de Ayuda para un mejor control de los cambios que impacten en los servicios. El catálogo de servicios es el punto de partida para la Mesa de Ayuda, para lo cual se ha propuesto también la definición del SLA referente a la atención de incidentes que permita una mejor gestión de los incidentes, pudiendo hacerse los ajustes necesarios que permitan evidenciar mejoras.

\subsubsection{Estrategias para mejorar la función de Mesa de Ayuda}

La Mesa de Ayuda tiene como principal objetivo entregar soporte a la provisión de servicios acordados, asegurando así la accesibilidad y disponibilidad de la organización de TI, además de desarrollar actividades de soporte. El presente 
artículo proporciona las opciones para que se pueda estructurar adecuadamente la Mesa de Ayuda. A continuación se muestran las características que pueden adecuarse a la ONGEI.

\section{Tabla 6. Tipo de estructuras organizacionales para la Mesa de Ayuda}

\begin{tabular}{|l|l|l|}
\hline \multicolumn{1}{|c|}{ Tipo de estructura } & \multicolumn{1}{|c|}{ Características } & \multicolumn{1}{c|}{ ONGEI } \\
\hline Mesa de ayuda local & $\begin{array}{l}\text { Localizada dentro de su comunidad } \\
\text { y puede existir para cada sede de } \\
\text { una empresa o entidad. }\end{array}$ & $\begin{array}{l}\text { No es adecuada, debido a } \\
\text { que generaría un incremento } \\
\text { innecesario en el costo. }\end{array}$ \\
\hline $\begin{array}{l}\text { Mesas de Ayuda } \\
\text { centralizada }\end{array}$ & $\begin{array}{l}\text { Estructura centralizada que usa una } \\
\text { mesa de servicios en una única } \\
\text { localización. }\end{array}$ & $\begin{array}{l}\text { Estructura más adecuada a la } \\
\text { realidad de la ONGEl. }\end{array}$ \\
\hline Mesa de Ayuda virtual & $\begin{array}{l}\text { Precisa del uso de tecnología a } \\
\text { través de Internet y podría cumplir } \\
\text { una función 24x7. }\end{array}$ & $\begin{array}{l}\text { De acuerdo al nivel de madurez } \\
\text { no es recomendable en este } \\
\text { momento, podría aplicarse poste- } \\
\text { rormente. }\end{array}$ \\
\hline Follow the sun & $\begin{array}{l}\text { Puede tener dos o más } \\
\text { mesas de servicios dispersas } \\
\text { geográficamente. Esto supone un } \\
\text { alto costo. }\end{array}$ & $\begin{array}{l}\text { No se aplicaría puesto que la } \\
\text { atención es a nivel nacional. }\end{array}$ \\
\hline
\end{tabular}

Elaboración propia

La estructura que se propone es la de Mesa de Ayuda centralizada, tal como se muestra en la figura 19, donde el único punto de contacto será el equipo de mesa de ayuda.

Figura 19. Estructura de la mesa de ayuda para ONGEI

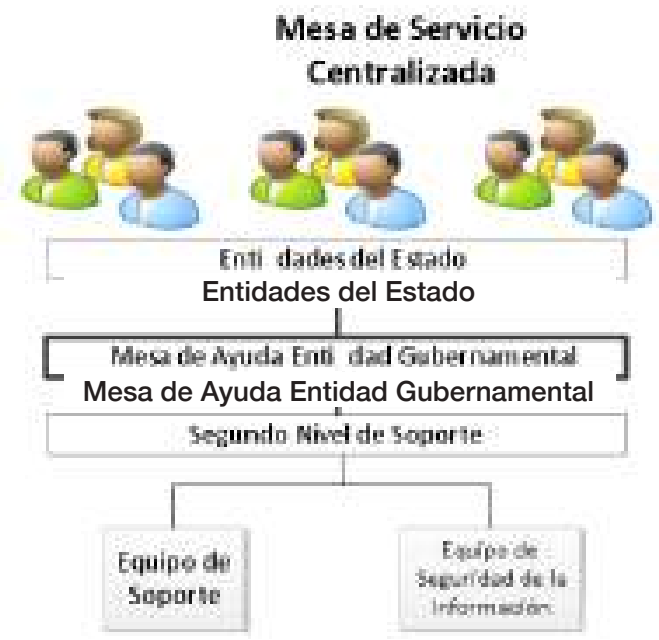

Elaboración propia 
5. Resultados de la investigación

5.1. Resultados del modelo de gestión de incidentes

\subsubsection{Incidentes registrados}

Los incidentes registrados durante el primer trimestre de 2015 disminuyeron en un $52,2 \%$ respecto al tercer trimestre de 2014 , debido al cumplimiento normativo de los sistemas Enriap y Plan Operativo Informático (POI), que establecen al mes de febrero como fecha límite para el cierre administrativo del año pasado. En comparación al mismo período del año 2014, se observa una disminución de 32,8 \%. Se observa una mejora gracias a una mejor gestión de los incidentes.

Tabla 7. Incidentes registrados en los trimestres de análisis

\begin{tabular}{|l|c|}
\hline \multicolumn{1}{|c|}{ Trimestre - Año } & Incidentes registrados \\
\hline Primer trimestre 2014 & 2318 \\
\hline Tercer trimestre 2014 & 3255 \\
\hline Primer trimestre 2015 & 1557 \\
\hline
\end{tabular}

Elaboración propia

5.1.2. Tiempos de mínimos y máximos tomados para resolver un incidente El tiempo mínimo para resolver un incidente se registró en mayo de 2015 (4 minutos), mientras que en el mes de abril del mismo año se registró el mayor tiempo máximo (3 horas y 10 minutos).

Tabla 8. Tiempos mínimos y máximos registrados

\begin{tabular}{|l|c|c|c|}
\hline Incidentes & $\begin{array}{c}\text { Tiempo mínimo } \\
\text { (minutos) }\end{array}$ & $\begin{array}{c}\text { Tiempo máximo } \\
\text { (horas:minutos) }\end{array}$ & $\begin{array}{c}\text { Promedio } \\
\text { (minutos) }\end{array}$ \\
\hline Marzo & 8 & $2: 00$ & 30 \\
\hline Abril & 5 & $3: 10$ & 45 \\
\hline Mayo & 4 & $2: 35$ & 20 \\
\hline
\end{tabular}

Fuente: Elaboración propia a partir de los resultados de la herramienta de gestión de incidentes. 
5.1.3. Incidentes derivados a equipos de trabajo clasificados por tipo de prioridad

Se observa, en cada uno de los meses en análisis, que la mayor cantidad de incidentes registrados tienen prioridad 4 y el equipo de mesa de ayuda es el que atiende más incidentes, seguido del equipo de soporte.

Figura 20. Incidentes por prioridad, de marzo a mayo de 2015

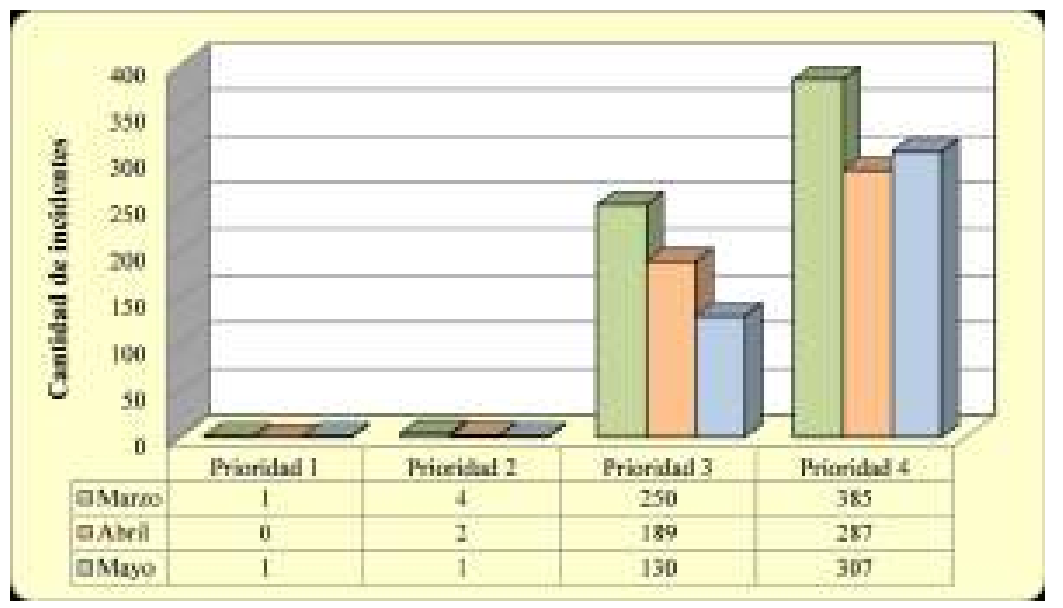

Elaboración propia

Figura 21. Incidentes por equipo de trabajo, de marzo a mayo de 2015

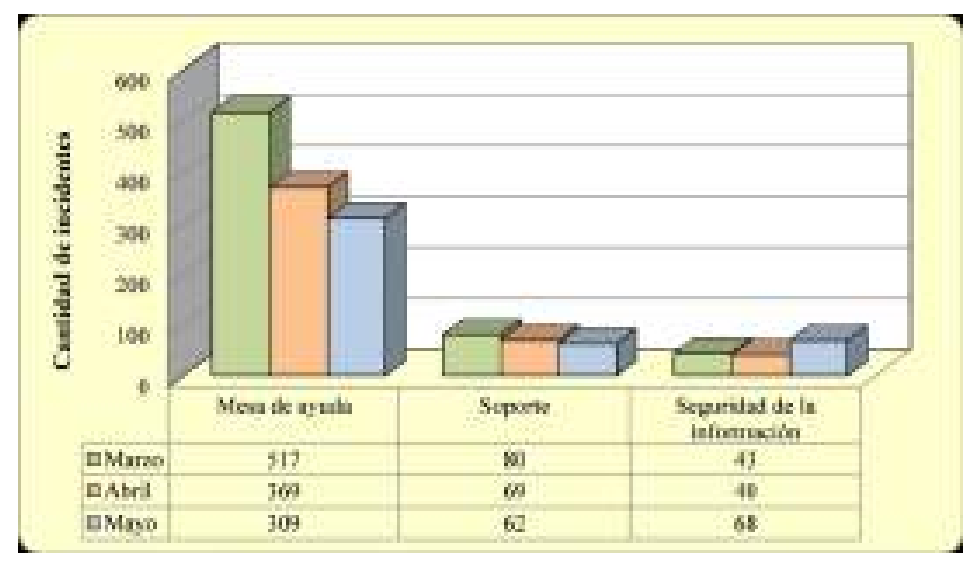

Elaboración propia 


\subsubsection{Incidentes que desembocaron en problemas}

Durante los meses de marzo, abril y mayo, los incidentes que desembocaron en problemas representaron el 9,7 \%, 5,0 \%, 3,4 \% respectivamente. Por lo tanto, más del 90 \% son incidentes resueltos por el equipo de mesa de ayuda, mostrándose una mejora constante.

\section{Tabla 9. Incidentes que desembarcaron en problemas}

\begin{tabular}{|c|c|c|c|}
\hline Mes-año & Total incidentes & $\begin{array}{c}\text { Cantidad de incidentes } \\
\text { problema }\end{array}$ & $\begin{array}{c}\text { Porcentaje de incidentes } \\
\text { problema }\end{array}$ \\
\hline mar-15 & 640 & 62 & $9.7 \%$ \\
\hline abr-15 & 478 & 24 & $5.0 \%$ \\
\hline may-15 & 439 & 15 & $3.4 \%$ \\
\hline
\end{tabular}

Elaboración propia

Validando esta información, se determinó que no todos los incidentes que desembocaron como problemas lo eran realmente, el personal está adaptándose a los cambios.

\subsection{Encuesta de satisfacción}

La muestra de entidades encuestadas fue de ciento veinte, siendo estas las que más usan los servicios de TI de la ONGEI. A continuación se muestran las preguntas utilizadas para la encuesta:

\section{Tabla 10. Cuestionario de preguntas}

\begin{tabular}{|c|c|}
\hline Objetivo & Pregunta \\
\hline $\begin{array}{l}\text { Comprobar si los procedimientos fueron } \\
\text { explicados de manera adecuada por } \\
\text { el personal de la ONGEl }\end{array}$ & $\begin{array}{l}\text { ¿Cómo califica las instrucciones que le dio la persona } \\
\text { de soporte para solucionar el incidente? }\end{array}$ \\
\hline Revisar tiempos de solución del incidente & $\begin{array}{l}\text { ¿Cómo califica el tiempo total utilizado para la solución } \\
\text { del incidente? }\end{array}$ \\
\hline $\begin{array}{l}\text { Comprobar la relación entre los equipos de } \\
\text { trabajo de ONGEl y los usuarios } \\
\text { de las entidades gubernamentales }\end{array}$ & $\begin{array}{l}\text { ¿Cómo fue la calidad en la atención que recibió del } \\
\text { personal de la Mesa de Ayuda? } \\
\text { ¿Cómo califica la nueva forma de atención de los } \\
\text { incidentes? }\end{array}$ \\
\hline
\end{tabular}

Calificación: Excelente (5), Muy Bueno (4), Bueno (3), Regular (2) y Deficiente (1). 
En la figura 22, se muestran los resultados de los meses de marzo, abril y mayo del 2015; en lo que se puede observar que el nivel de aprobación se encuentra alrededor del 70 \% (división del promedio de la puntuación y el puntaje máximo de aceptación); lo que indica que se ha percibido cierto nivel de mejora pero precisa mejorar más. Cabe resaltar que es la primera vez que se realiza una encuesta de satisfacción en la ONGEl.

\section{Figura 22. Resultado de la encuesta dirigida a los usuarios}

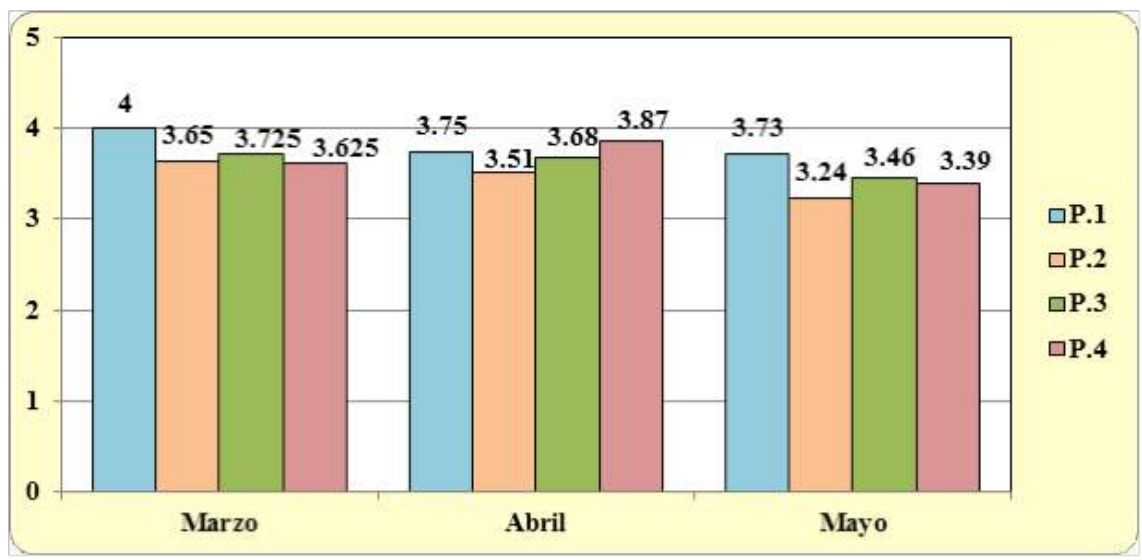

Elaboración propia

\subsection{Análisis de la investigación}

Los puntos favorables de la implementación fueron los siguientes:

- Las buenas prácticas para la atención y registro de incidentes fueron bien recibidas.

- El equipo de mesa de ayuda es el único punto de contacto además de ser el primer nivel de resolución de los incidentes.

- Los procesos propuestos para el monitoreo de los servicios críticos fueron aprobados y se comenzó a registrar eventos en la herramienta ServiceTonic, para así obtener tendencias y realizar la detección temprana de incidentes y problemas.

- Las entidades usuarias percibieron las mejoras pero aún creen que hay temas por efectuar, de acuerdo a la encuesta de satisfacción.

- A nivel gerencial fue aceptado el hecho de tener una línea base de indicadores que permita hacer los ajustes necesarios para la mejora continua. 
También se encontraron algunos inconvenientes:

- $\quad$ Si bien a nivel gerencial y de jefatura fue bien recibido, por parte del nivel operativo algunas personas se mostraron reacias al cambio a pesar de que en un principio estuvieron de acuerdo con la propuesta.

- Algunas entidades del estado siguieron con la forma tradicional de notificación de los incidentes. Por ser esta una etapa piloto fue aceptada, pero se les informó cómo debía ser la forma de notificación la próxima vez.

\section{Conclusiones y recomendaciones}

\subsection{Conclusiones}

i. El presente trabajo permitió ordenar y clasificar mejor los incidentes, el cual evita la duplicidad de registros y el reprocesamiento. Luego de la implementación se observó una disminución de más del 50 \% de incidentes.

ii. La aplicación del modelo propuesto involucró cambios tecnológicos y de organización en la ONGEl, que implicó cambiar los hábitos de trabajo para la atención de incidentes, teniendo un solo punto de registro y un nuevo nivel de coordinación entre equipos de trabajo. Si bien el modelo muestra mejoras, no fue aceptado del todo por algunas personas que se mostraron reacias al cambio.

iii. El monitoreo temprano permitió disminuir la carga de trabajo del equipo de mesa de ayuda, ya que se pudieron detectar tempranamente alertas y eventos comunes como es el caso de bloqueo de usuario o bloqueo de usuarios en el sistema de mensajes SMS. Si bien la parte de gestión de eventos no está implementada, estos procesos preventivos nos muestran que es necesaria su implementación.

iv. Se logró establecer una línea base de indicadores que permitirán el monitoreo de la gestión de incidentes y realizar los ajustes necesarios.

\subsection{Recomendaciones}

i. Optimizar la gestión de problemas, con el fin de documentar mejor los problemas resueltos y entregar servicios de más calidad.

ii. Formalizar el proceso gestión de eventos para la detección temprana de incidentes y problemas que puedan presentarse.

iii. Crear un plan de continuidad para el personal técnico de la ONGEl, con el propósito de evitar la pérdida de talento humano. Se propone crear contratos de mediano y 
largo plazo para la continuidad del personal y evitar salidas bruscas ante cambios directivos.

iv. Continuar con las capacitaciones a los equipos de trabajo de la ONGEl para lograr un nivel de especialización mayor en ITIL que genere un ambiente en el cual se sigan las buenas prácticas de ITIL. De esta manera, se podrá obtener el nivel de madurez que permita atender mejor las necesidades de los usuarios.

\section{Referencias}

Bon, J., De Jong, A., Kolthof, A., Pieper, M, Tjassing, R., Van der Veen, A., Verheijen, T. (2008). Gestión de servicios TI basado en ITIL. (Vol. 3). Zaltbommel: Van Haren Publishing. Recuperado de http://www.vanharen.net/ Samplefiles/9789087531065_gesti-n-de-serviciosti-basado-en-itil-v3-guia-de-bolsillo.pdf

Office of Government Commerce (2007). ITL V3 Service Operation. London: The Stationery Office.

Oficina Nacional de Gobierno Electrónico e Informática (2012). Gobierno electrónico y su contribución en el proceso de modernización y descentralización de la gestión del estado. Lima: Autor

Osiastis S.A. (s.f). Gestión de incidentes. Proceso. Recuperado de Itil.osiatis.es/Curso_ITL/ Gestion_Servicios_Tl/gestion_de_incidentes/proceso_gestion_de_incidentes/proceso_ gestion_de_incidentes.php

Perú. Presidencia del Consejo de Ministros. (2003). Decreto Supremo N. ${ }^{\circ}$ 066-2003-PCM. Lima, Perú. Recuperado de http://www.peru.gob.pe/docs/planes/4/plan_4_ 2016_decreto_supremo_n_066_2003_pcm_fusion_sji_a_pcm.pdf

Perú. Presidencia del Consejo de Ministros. (2003). Decreto Supremo N. ${ }^{\circ}$ 067-2003-PCM. Lima, Perú. Recuperado de http://www.ongei.gob.pe/normas/0/norma_O_decreto\%20 supremo\%20n\%c2\%ba\%20067-2003-pcm.pdf

Tang, X., Todo, Y. (2013). A Study of Service Desk Setup in Implementing IT Service Management in Enterprises. Technology and Investment, 4(3), 190-196. DOl: 10.4236/ti.2013.43022

United Nations. Department of Economic and Social Affairs. (2012). E-Government Survey 2012: e-Government for The People. Nueva York: United Nations.

United Nations. Department of Economic and Social Affairs. (2014). E-Government Survey 2014: e-Government for the Future We Want. Nueva York: United Nations. 
Yurivilca, C. y Loayza C. (2014). Manual de gestión de la ONGEl. Versión 1.0. Lima: ONGEl (no publicado).

\section{Glosario}

- Acuerdo: Documento que permite y describe el entendimiento entre dos partes.

i. Acuerdo de nivel de servicio - ANS (Service Level Agreement - SLA)

ii. Acuerdo de nivel operativo (Operational Level Agreement - OLA)

iii. Acuerdo entre el área de TI y otra área de la organización.

- La CMDB (Configuration Management Data Base): Base de datos que tiene registros de los elementos de configuración que se asocian con la infraestructura de TI.

- Incidente: Interrupción no planificada de un servicio de TI o reducción en su calidad.

- Solución temporal (workaround): Reducción o eliminación del impacto de un incidente o problema para el que la solución definitiva aún no se encuentra disponible.

- Sistema bitácora: Software desarrollado por una empresa externa. Este software fue desarrollado a medida que se registraban inicialmente los incidentes.

- Urgencia: Medida del tiempo en que un incidente, problema o cambio tendrá un impacto significativo para el negocio. 